\title{
Determinação de anti-inflamatórios na água e sedimento e suas relações com a qualidade da água na bacia do Alto Iguaçu, Curitiba-PR.
}

Determination of anti-inflammatory in water and sediment and its relations with water quality in Alto Iguaçu watershed, Curitiba-PR

Rafael Duarte Kramer ${ }^{1}$; Alinne Mizukawa ${ }^{2}$; Alessandra Honjo Ide ${ }^{3}$; Luana Oliveira Marcante ${ }^{4}$;

Maruricius Marques dos Santos ${ }^{5}$ e Júlio César Rodrigues de Azevedo ${ }^{6}$

\author{
${ }^{1,2}$ Programa de Pós-Graduação em Engenharia de Recursos Hídricos e Ambiental (PPGERHA), Universidade Federal do Paraná, Curitiba, PR, Brasil \\ rafaelkramer@outlook.com; alimizu@yahoo.com.br \\ 3,4,5,6 Laboratório de Estudos Avançados em Química Ambiental (LEAQUA), Universidade Tecnológica Federal do Paraná, Curitiba, PR, Brasil \\ alessandrahide@hotmail.com; luana_tgambiental@yahoo.com.br; mauricius_mds@hotmail.com; jcrazevedo@utfpr.edu.br
}

Recebido: 10/11/14 - Revisado: 01/02/15 - Aceito: 18/05/15

\section{RESUMO}

A contaminação ambiental por poluentes emergentes tem sido explorada em diferentes pesquisas na área ambiental. Isso deve, em grande parte, pela baixa eficiência dos tratamentos de efluentes municipais e pelo alto consumo de produtos quimicos. A bacia bidrográfica do Alto Iguacu, localizada na Região Metropolitana de Curitiba, apresenta alta densidade populacional em seu entorno e, consequentemente, problemas na qualidade da água de seus rios. Considerando este aspecto, o presente estudo abrangeu a avaliação de contaminantes emergentes em cinco rios pertencentes a esta bacia. Foi avaliada a contaminação pelos anti-inflamatórios ibuprofeno, paracetamol e diclofenaco, na água superficial e nos sedimentos, durante um ano, totalizando quatro campanbas amostrais. O ibuprofeno foi quantificado somente na primeira amostragem, apresentando concentrações elevadas no rio Belém (729 ng. $\left.\mathrm{L}^{-1}\right)$. Já o diclofenaco foi amplamente detectado na água, chegando a concentrações de $285 \mathrm{ng} . \mathrm{L}^{-1}$ na terceira coleta no rio Iguaçu. Ao contrário dos demais, o paracetamol foi frequentemente quantificado na água, tendo os rios Iguaçu e Belém com mais presença deste fármaco, o qual também foi determinado nos sedimentos, especialmente no rio Barigui, chegando a apresentar $6,896 \mu \mathrm{g} g^{-1}$. A presença desses compostos nos rios amostrados confirma a interferência antrópica que a bacia do Alto Iguaçu sofre da desordenada urbaniz̧ação.

Palavras Chave: Diclofenaco. Ibuprofeno. Paracetamol. Esgoto doméstico. Região Metropolitana de Curitiba

\begin{abstract}
Environmental contamination by emerging pollutants has been explored in different studies in the environmental area. This is due, in the most part, by the low efficiency of municipal wastewater treatment and the high chemical consumption. The watershed of the Alto Iguacu located in the Metropolitan Region of Curitiba has a high population density in your surroundings and, consequently, has some problems in water quality of its rivers. Considering this aspect, this study comprehended the evaluation of emerging contaminants in five rivers inside this watershed. Contamination by anti-inflammatory ibuprofen, paracetamol and diclofenac in surface water and sediment was evaluated for a year, a total of four sampling campaigns. Ibuprofen was found only in the first sampling, but in high concentrations in the Belémriver $\left(729 \mathrm{ng} . \mathrm{L}^{-1}\right)$. Diclofenac had been widely detected in water and it reached concentrations of $285 \mathrm{ng} . \mathrm{L}^{-1}$ in the third sampling in the Iguacu river. Unlike the rest, paracetamol was often quantified in the water, being the Iguacu and Belém rivers with more presence of this drug. It was also determined in sediments, especially in Bariguiriver, being founded 6,896 $\mu \mathrm{g} \mathrm{g}^{-1}$. The presence of these compounds in the sampled rivers confirms an anthropogenic interference with the Alto Iguacu watershed, which suffers from unplanned urbanization.
\end{abstract}

Keywords: Diclofenac. Ibuprofen. Paracetamol. Domestic sewage. Metropolitan Region of Curitiba 


\section{INTRODUÇÃO}

A matéria orgânica dissolvida (MOD) apresenta, em sua composição, uma complexa mistura de matéria orgânica natural (alóctone ou autóctone) ou de origem antropogênica, como esgotos domésticos (AHMAD; REYNOLDS, 1995; MA; ALLEN; YIN, 2001; OLIVEIRA et al., 2006).

A MOD, inicialmente, pode ser avaliada através das técnicas de espectroscópica: emissão de fluorescência molecular ou absorção na região do ultravioleta e visível (CHEN et al., 2002; KNAPIK; FERNANDES; AZEVEDO, 2014; OLIVEIRA et al., 2006; PEURAVUORI; KOIVIKKO; PIHLAJA, 2002; SENESI, 1990). No estudo da MOD, na emissão da fluorescência é possível destacar os espectros de emissão com excitação ( $\lambda \mathrm{Exc}$ ) em 370nm e determinação da emissão ( $\lambda \mathrm{Em})$ em 450 e $500 \mathrm{~nm}$ (OLIVEIRA et al., 2006; WESTERHOFF; ANNING, 2000). Também são aplicadas as técnicas de excitação, de espectros sincronizados e o de matriz excitação-emissão (AZEVEDO; NOZAKI, 2008; CHEN et al., 2002; MA; ALLEN; YIN, 2001; OLIVEIRA et al., 2006; PEURAVUORI; KOIVIKKO; PIHLAJA, 2002; SENESI, 1990).

Após identificar as possíveis fontes da matéria orgânica (autóctone, alóctone pedogênica ou alóctone antropogênica) pôde ser realizado um estudo mais detalhado dos compostos orgânicos presentes no ambiente aquático, como a determinação de contaminantes emergentes.

Contaminantes emergentes estão relacionados a qualquer substância química presente no nosso cotidiano como fármacos, produtos de higiene, produtos de uso veterinário, embalagens de alimentos, agroquímicos, retardantes de chama, hormônios sexuais femininos, entre outros, que possam ser encontrados em matrizes ambientais e biológicas em concentrações muito baixas, da ordem de ng.L-1 (AQUINO; BRANDT; CHERNICHARO, 2013). Normalmente, não são monitoradas ou não possuem ainda legislação, sendo que estas substâncias, muitas vezes, podem afetar o sistema endócrino, apresentando risco potencial à saúde (LA FARRE et al., 2008).

Dentro das principais classes dos poluentes emergentes podemos citar os produtos farmacêuticos. O grande consumo de medicamentos pela população mundial tem fomentado pesquisas sobre contaminação por diferentes princípios ativos e metabólitos provenientes dos fármacos (KASPRZYK-HORDERN; DINSDALE; GUWY, 2009), pois estes podem estar presentes nos ambientes aquáticos. O Brasil é o quarto maior consumidor mundial de medicamentos, com consumo médio de 11 caixas de diferentes fármacos por pessoa/ano (EICKHOFF; HEINECK; SEIXAS, 2009). Esses produtos farmacêuticos, provavelmente, chegam ao meio ambiente, não só por causa do uso indevido, mas também em virtude do uso cotidiano e descarte inapropriado.

Desenvolvidas para um fim terapêutico, as estruturas químicas dos medicamentos tendem a ser persistentes no ambiente, sendo que, aproximadamente $75 \%$ de algumas classes de fármacos são excretadas pelo corpo humano e animal sem sofrerem alterações (KUMMERER, 2010). A outra parte é metabolizada, transformando-se em outros compostos (metabólitos), podendo ter uma alteração das suas características e até mesmo uma maior acumulação no ambiente (KASPRZYK-HORDERN, 2010). Há uma preocupação com o uso excessivo de fármacos, porém esses medicamentos, quando não são utilizados, quase sempre são descartados através de vasos sanitários, escoados com a chuva ou tratados como resíduos sólidos comuns, não seguindo um procedimento de descarte adequado (KUMMERER, 2010).

Outra forma de entrada dos contaminantes emergentes, em especial fármacos e seus metabólitos, no ambiente é através de várias fontes pontuais e não pontuais tais como: plantas de fabricação; efluentes das Estações de Tratamento de Esgoto e/ ou industriais; resíduos domésticos; aterros sanitários e excreção animal (DAUGHTON; TERNES, 1999). Uma quantidade significativa dos fármacos originais e seus metabólitos são excretados na urina, fezes ou esterco animal e, posteriormente, chegam às Estações de Tratamento de Esgoto (ETE's) para serem degradados (ONESIOS; YU; BOUWER, 2009). Há três rotas principais para os medicamentos nas ETE's (RICHARDSON; BOWRON, 1985): pode ser mineralizado a gás carbônico e água (biodegradado), como a biodegradação do ácido acetilsalicílico; pode ser metabolizado ou ser degradado parcialmente, como as penicilinas; ou então ser persistente, como os clofibratos. Apesar de existirem modernas ETE's, estudos tem comprovado que diferentes compostos orgânicos de interesse ambiental não são biodegradados ou eliminados completamente por estes procedimentos de tratamento, persistindo então nos sistemas aquáticos em que os efluentes são lançados (SCHNELL et al., 2009).

O caminho percorrido por um fármaco no meio ambiente, desde a sua produção até sua total degradação, ainda é desconhecida (FATTA-KASSINOS; MERIC; NIKOLAOU, 2011). Os compartimentos ambientais com maior incidência de contaminação são o sedimento e a água, tanto superficial como subterrânea (KASPRZYK-HORDERN; DINSDALE; GUWY, 2009). A possível presença de compostos farmacêuticos em águas superficiais e subterrâneas e nos sedimentos acarreta na necessidade de mais estudos que determinem suas concentrações e seus efeitos tóxicos no meio ambiente (SILVA et al., 2011).

Uma das classes de produtos farmacêuticos muito presente no nosso cotidiano é a dos anti-inflamatórios, que consiste, basicamente, em um grupo de compostos ativos com finalidade de controlar a inflamação, promover a analgesia e combater a hipertermia. Grandes quantidades de analgésicos são prescritas em atendimento médico humano, mas geralmente são vendidos em quantidades muito mais elevadas e sem receita (SHIN; OH, 2012). No Brasil, os anti-inflamatórios não-esteroidais (AINEs) são medicamentos que não precisam de prescrições médicas para ser comprados e fazem parte da composição de diversos medicamentos à venda, caracterizando assim esta classe de medicamentos como a mais prescrita por médicos e dentistas e, consequentemente, uma das mais consumidas (EMERENCIANO et al., 2008). Nesta extensa classe existem: ácido acetilsalicílico, cetoprofeno, fenoprofeno, flurbiprofeno, ácido tiaprofenico, carprofeno, loxoprofeno, indometacina, ibuprofeno, diclofenaco e acetaminofeno (paracetamol).

Não muito distante dessa problemática ambiental, a cidade de Curitiba vem sofrendo com alguns danos em seus corpos hídricos. O aumento populacional da região metropolitana de Curitiba (RMC), que hoje representa aproximadamente 25\% da 
Kramer et al.:Determinação de anti-inflamatórios na água e sedimento e suas relações com a qualidade da água na bacia do Alto Iguaçu, Curitiba-PR.

população total do estado, causou uma ocupação desordenada em áreas de mananciais que abastecem a região, prejudicando a disponibilidade e qualidade hídrica (YAMAMOTO; CANALI, 2012). A RMC está inserida na bacia do Alto Iguaçu e os impactos causados pela população já estão sendo refletidos nas águas de abastecimento de milhões de pessoas delas servidas (ANDREOLI et al., 2000).

O rio Iguaçu, o principal rio da bacia hidrográfica em questão, ao longo de sua trajetória pela bacia do Alto Iguaçu recebe as cargas dos rios Belém, Barigui, Atuba, Iraí, Palmital, entre outros, diminuindo consideravelmente a qualidade da água. Muitos desses rios cortam a cidade de Curitiba e lançam toda a sua carga proveniente da poluição difusa, efluentes domésticos, lançamentos pontuais e efluentes resultantes das indústrias na bacia do Alto Iguaçu (KNAPIK et al., 2008). A principal carga poluidora na RMC está relacionada aos esgotos domésticos. $\mathrm{O}$ serviço de coleta de esgoto não atende a todas as sub-bacias e quando atende possui algumas falhas na rede ou na eficiência do tratamento. Mesmo algumas regiões, tendo a rede de coleta de esgoto, muitas casas são ligadas erroneamente na rede de águas pluviais ou são lançados diretamente nos rios na forma in natura (YAMAMOTO; CANALI, 2012).

Neste estudo, foram realizadas amostragens da água superficial e sedimento dos rios Atuba, Barigui, Belém, Iraí e Iguaçu, na Região Metropolitana de Curitiba, com a finalidade de verificar possíveis contaminações no meio ambiente por diclofenaco (DIC), ibuprofeno (IBU) e paracetamol (PAR).

\section{METODOLOGIA}

\section{Área de estudo}

O presente estudo foi desenvolvido na Bacia do Alto
Iguaçu que está situada na Região Metropolitana de Curitiba (RMC). A Bacia do Alto Iguaçu abrange 30 sub-bacias, contemplando uma área total de aproximadamente $2881,73 \mathrm{~km}^{2}$ (SEMA, 2010). Foram realizadas quatro amostragens: abril, junho, setembro e novembro de 2011; foram coletados amostras de água superficial e de sedimento de 11 pontos em 5 diferentes rios da RMC, todos pertencentes à bacia hidrográfica do Alto Iguaçu: rio Belém; rio Barigui; rio Atuba; rio Iraí e rio Iguaçu (Figura 1). Na Tabela 1, encontram-se as coordenadas e as siglas usadas dos pontos amostrados.

Tabela 1 - Localização dos pontos amostrados no estudo

\begin{tabular}{clcc}
\hline Rio & Latitude (S) & Longitude (O) & $\begin{array}{c}\text { Sigla do } \\
\text { ponto }\end{array}$ \\
\hline Belém & $25^{\circ} 26^{\prime} 51.93^{\prime \prime}$ & $49^{\circ} 15^{\prime} 04.10^{\prime \prime}$ & BL1 \\
\hline Belém & $25^{\circ} 30^{\prime} 26.58^{\prime \prime}$ & $49^{\circ} 12^{\prime} 54.22^{\prime \prime}$ & BL2 \\
\hline Barigui & $25^{\circ} 25^{\prime} 34.43^{\prime \prime}$ & $49^{\circ} 18^{\prime} 24.66^{\prime \prime}$ & BA1 \\
\hline Barigui & $25^{\circ} 25^{\prime} 52.46^{\prime \prime}$ & $49^{\circ} 18^{\prime} 47.51^{\prime \prime}$ & BA2 \\
\hline Barigui & $25^{\circ} 27^{\prime} 54.09^{\prime \prime}$ & $49^{\circ} 19^{\prime} 12.81^{\prime \prime}$ & BA3 \\
\hline Barigui & $25^{\circ} 30^{\prime} 53.21^{\prime \prime}$ & $49^{\circ} 20^{\prime} 18.85^{\prime}$ & BA4 \\
\hline Atuba & $25^{\circ} 27^{\prime} 53.73^{\prime \prime}$ & $49^{\circ} 11^{\prime} 25.56^{\prime}$ & AT1 \\
\hline Atuba & $25^{\circ} 28^{\prime} 25.76^{\prime \prime}$ & $49^{\circ} 11^{\prime} 07.87^{\prime \prime}$ & AT2 \\
\hline Iguaçu & $25^{\circ} 29^{\prime} 03.73^{\prime \prime}$ & $49^{\circ} 11^{\prime} 25.011^{\prime}$ & IG1 \\
\hline Iguçu & $25^{\circ} 31^{\prime} 42.10^{\prime \prime}$ & $49^{\circ} 13^{\prime} 08.50^{\prime \prime}$ & IG2 \\
\hline Iraí & $25^{\circ} 27^{\prime} 16.61^{\prime \prime}$ & $49^{\circ} 10^{\prime} 17.11^{\prime \prime}$ & IR1 \\
\hline
\end{tabular}

\section{Análises físicas e químicas}

A amostragem em campo, em alguns pontos, foi realizada em pontes altas, utilizando garrafa de Van Dorn de 5 litros para auxiliar nas coletas de água e tomado o cuidado de realizar amostragem na superfície da água. Foram coletados 1,5 L de água superficial dos rios para a realização das análises físicas e químicas e as amostras foram preservadas com gelo até a chegada ao laboratório. Em campo, foram obtidos os valores

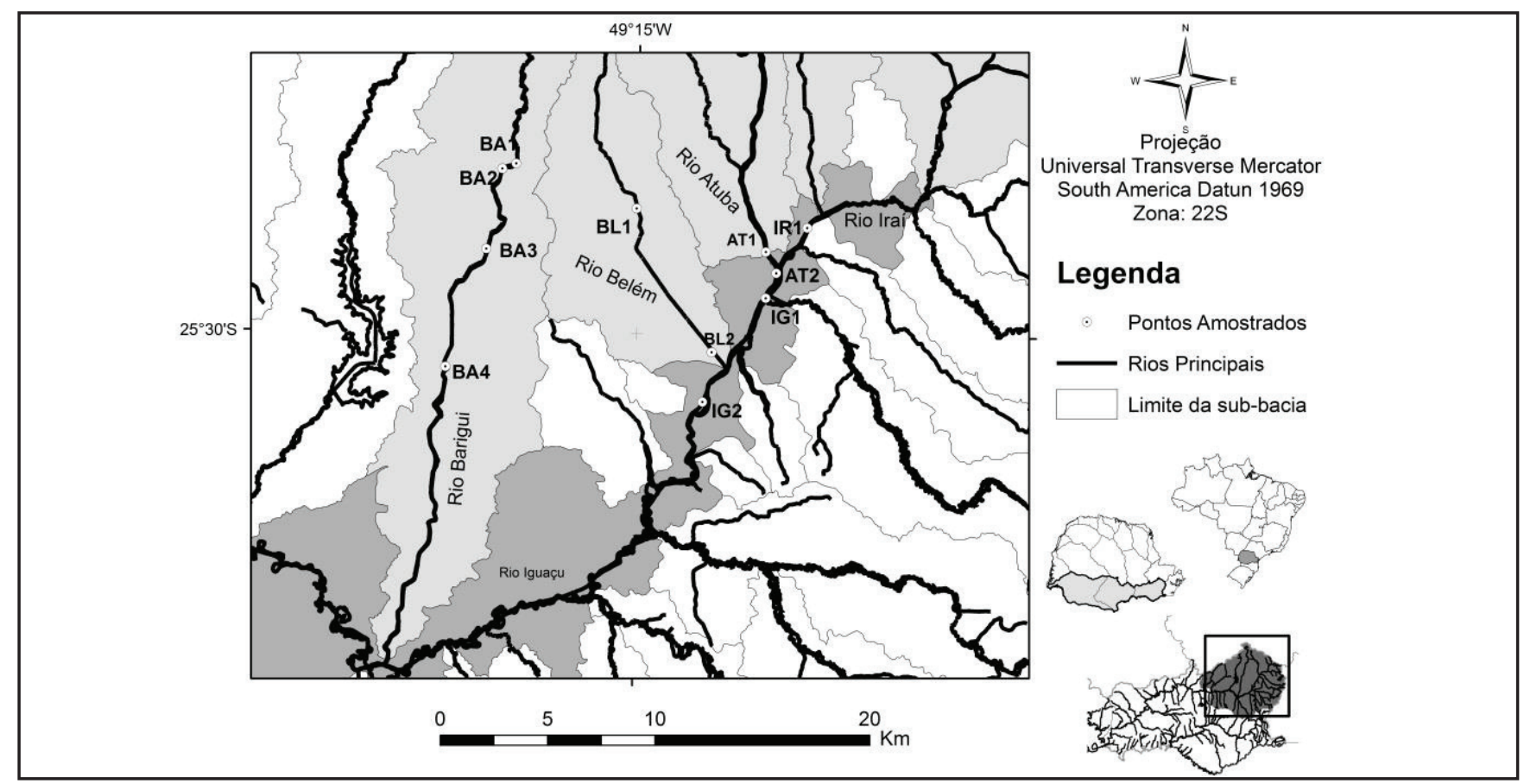

Figura 1 - Indicação da localização dos pontos de amostragem na Bacia do Alto Iguaçu 
da temperatura da água $\left({ }^{\circ} \mathrm{C}\right)$, do oxigênio dissolvido $\left(\mathrm{mg} \cdot \mathrm{L}^{-1}\right)$, do $\mathrm{pH}$, da condutividade elétrica $\left(\mu \mathrm{S} . \mathrm{cm}^{-1}\right)$, do potencial redox $(\mathrm{mV})$ e dos sólidos totais dissolvidos $\left(\mathrm{mg}^{-\mathrm{L}^{-1}}\right)$, todos com a sonda multiparâmetro Hanna Instruments. A turbidez (NTU) foi estimada utilizando um turbidímetro digital Hanna. Os níveis de precipitação foram obtidos com o Sistema Meteorológico do Paraná (SIMEPAR) das subestações de Curitiba e Pinhais.

Para realização das análises físicas e químicas, as amostras foram filtradas com membranas Millipore de éster de celulose, 0,45 $\mu \mathrm{m}$. Foram analisados: o $\mathrm{N}$-nitrito (método colorimétrico), o N-nitrato (método da redução do Cádmio), o N-amoniacal (método de fenato), o ortofosfato (método do ácido ascórbico) e, nas amostras in natura, foi quantificada a concentração de fósforo total (digestão ácida) APHA (2012).

As análises de espectroscopia na região do ultravioleta e visível foram realizadas no dia da campanha com a fração dissolvida $(0,45 \mu \mathrm{m})$. Foram obtidos espectros na faixa de 200 a $700 \mathrm{~nm}$ com o espectrofotômetro Cary 50 - Varian, utilizando-se cubeta de quartzo, com caminho ótico de 1,0 cm. Como branco, foi utilizado água ultrapura.

A análise por fluorescência molecular foi realizada em equipamento Varian Cary Eclipse Fluorescence Spectrophotometer, utilizando lâmpada de xenônio e voltagem de $900 \mathrm{~V}$. Foram obtidos espectros de emissão, com excitação em 370 nm. Também foram obtidos espectros de varredura sincronizada, com excitação de 250 a $600 \mathrm{~nm}(\Delta \lambda=18 \mathrm{~nm})$. Nos espectros de MEE, as medidas foram realizadas nos comprimentos de onda de 250 a $600 \mathrm{~nm}$ para excitação e de 250 a $700 \mathrm{~nm}$ para emissão, onde foram determinados alguns picos principais (T1, T2, A, B C), segundo Chen et al. (2003). Todos os espectros de fluorescência foram obtidos aplicando-se $240 \mathrm{~nm} \mathrm{~min}{ }^{-1}$, fenda de $5 \mathrm{~nm}$, cubeta de quartzo de $1 \mathrm{~cm}$ e água ultra pura como branco. Determinações do espectro de água ultrapura foram realizadas para um controle do sinal analítico e eliminação do espalhamento Raman dos espectros.

O carbono orgânico dissolvido (COD) $\left(\mathrm{pH}<2, \mathrm{H}_{2} \mathrm{SO}_{4}\right)$ foi determinado empregando o equipamento Hiper TOC Thermo Scientific, aplicando $10000 \mathrm{C}$ na combustão da amostra.

\section{Extração dos fármacos}

Foi coletado $1 \mathrm{~L}$ de amostra de água superficial de cada ponto com o auxílio de uma garrafa de Van Dorn e armazenados em frascos âmbar. Esses foram previamente descontaminados com $\operatorname{Extran}^{\circledR}$ e, posteriormente, aquecidos a $450{ }^{\circ} \mathrm{C}$ durante 3 horas. As amostras foram filtradas com membrana $0,45 \mu \mathrm{m}$ e, em seguida, adicionado $\mathrm{HCl}$ 6,0 mol.L $\mathrm{L}^{-1}$ até o $\mathrm{pH}$ ser próximo de 3 .

A extração dos fármacos, nas amostras com o pH ajustado, foi realizada empregando a técnica de extração em fase sólida (SPE), utilizando cartuchos de C18. As amostras foram passadas a uma velocidade de 8 a $10 \mathrm{~mL} \cdot \mathrm{min}^{-1}$. Os cartuchos foram secados no fluxo de nitrogênio. A eluição foi realizada $\operatorname{com} 20 \mathrm{~mL}$ de acetonitrila e rotaevaporados a $40^{\circ} \mathrm{C}$ e, o conteúdo foi redissolvido em $1 \mathrm{~mL}$ de metanol com o auxílio de um ultrassom e transferidos para vials de $2 \mathrm{~mL}$.

A derivatização dos fármacos extraídos foi realizada da seguinte forma: os vials foram colocados em estufa a $30^{\circ} \mathrm{C}$ para total evaporação do solvente e posterior dissolução em $0,93 \mathrm{~mL}$ de acetato de etila e adição de $0,05 \mathrm{~mL}$ de um padrão previamente conhecido para fortificação do sinal analítico. Por final, a amostra foi derivatizada com $0,02 \mathrm{~mL}$ de BSTFA $+1 \%$ de TMCS em estufa a $60^{\circ} \mathrm{C}$ durante 30 minutos. Posteriormente, as amostras foram analisadas por cromatografia gasosa (CG-FID).

As amostras de sedimento foram coletadas com o auxílio da draga de Petersen modificada e preservadas a temperatura de $-20^{\circ} \mathrm{C}$. Foram realizadas 3 campanhas de amostragem (abril, junho e novembro de 2011) de sedimento que coincidiram com as amostragens da água. As análises do sedimento seguiram parcialmente a metodologia descrita por Xu et al. (2008). A análise foi realizada em $5 \mathrm{~g}$ de sedimento. Foram realizadas 4 extrações sucessivas com dois diferentes solventes, sendo: as duas primeiras com $5 \mathrm{~mL}$ de acetona e as duas últimas com $5 \mathrm{~mL}$ de acetato de etila. Em cada adição de solvente, a amostra foi sonificada durante 10 minutos e depois centrifugada durante 10 minutos, a $4000 \mathrm{rpm}$. O sobrenadante resultante foi acumulado e, no final, rotaevaporado a $40{ }^{\circ} \mathrm{C}$ até a secura. Foram adicionados $3 \mathrm{~mL}$ de metanol para a reconstituição da amostra e avolumados para 25 $\mathrm{ml}$ com água ultra pura. A etapa seguinte consistiu na extração, em fase sólida, igualmente à utilizada na extração das amostras de água superficial, tal como uma etapa de limpeza (clean up) da amostra extraída do sedimento.

\section{Cromatografia gasosa}

Para as análises, foi utilizado um cromatógrafo de fase gasosa (CG) com um detector de ionização por chama (FID) (Varian 450-GC) e amostrador automático (Varian CP-8410). A separação cromatográfica foi feita com uma coluna capilar HP-5MS, $30 \mathrm{~m}$ x 0,25 mm, 5\% difenil-dimetilpolissiloxano $(0,25 \mu \mathrm{m})$. O modo Split/Splitless foi usado com o gás de arraste nitrogênio com um fluxo de $1 \mathrm{~mL} \cdot \mathrm{min}^{-1}$. As temperaturas do injetor e detector foram fixadas em $250^{\circ} \mathrm{C}$. O programa de temperatura do forno foi: temperatura inicial de $70^{\circ} \mathrm{C}$ durante 1 minuto, subindo para $120^{\circ} \mathrm{C}$ a uma taxa de $20^{\circ} \mathrm{C} \mathrm{min}^{-1}$, depois foi a $250^{\circ} \mathrm{C}$ na taxa de $10^{\circ} \mathrm{C} \mathrm{min}^{-1}$ e foi a $270^{\circ} \mathrm{C}$ em uma taxa

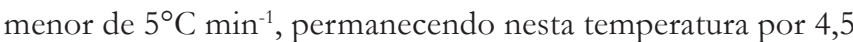
minutos, totalizando uma corrida de 25 minutos. Foi injetado no equipamento $1 \mu \mathrm{L}$ de amostra.

\section{Análises estatísticas}

Foi utilizado teste não paramétrico de correlação de Sperman pelo fato da não homogeneidade dos dados. Os dados de censura foram considerados a metade do limite de detecção (LD/2) (CHRISTOFARO; LEAO, 2014).

\section{RESULTADOS E DISCUSSÃO}

$\mathrm{Na}$ Figura 2, constam os valores dos índices de precipitação na região de Curitiba e a indicação das campanhas realizadas.Os rios Atuba e Iraí encontram-se em Pinhais e os demais pontos dos rios (Belém, Barigui, Iguaçu) foram amostrados em Curitiba. A diferença de precipitação entre as cidades 


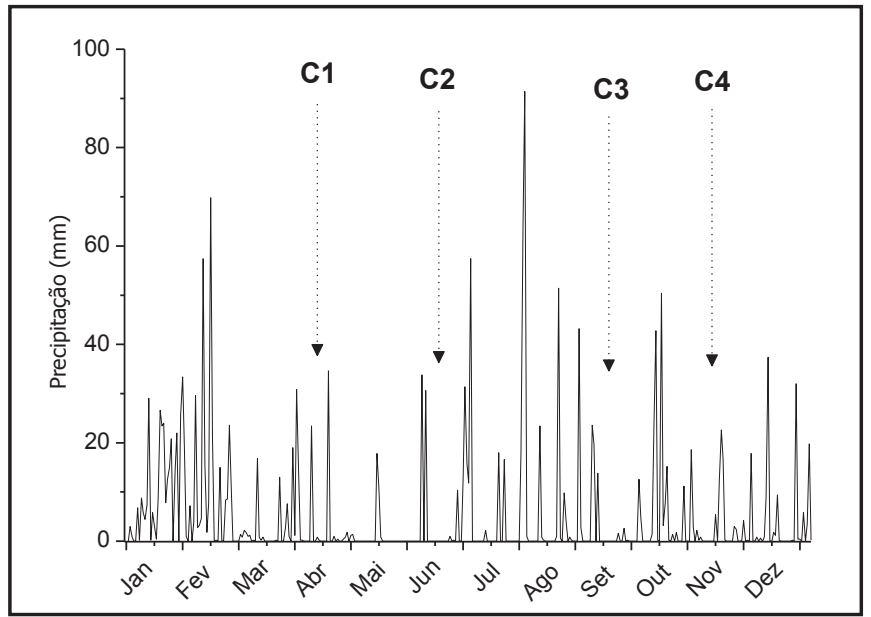

Figura 2 - Níveis de precipitação, em mm, próximos dos locais amostrados. Onde $\mathrm{C} 1$ refere-se à primeira, $\mathrm{C} 2$ à segunda, $\mathrm{C} 3$ à terceira e $\mathrm{C} 4$ à quarta campanha

nos períodos amostrados foi pequena e apenas na primeira amostragem (C1) houve menor precipitação em Curitiba do que em Pinhais. Considerando a média das chuvas de sete dias antes das amostragens, a segunda campanha (C2) apresentou-se com chuvas mais intensas (Curitiba, 64,4 mm e Pinhais, 67,8 mm) e a quarta (C4) foi a menos intensa.

Na tabela 2, constam os valores, considerando a média de todos os pontos amostrados em cada campanha, no respectivo rio. Por esta razão, os desvios padrões podem apresentar valores mais elevados, pois existem pontos no rio que apresentam menor concentração em relação a outro ponto na mesma campanha, ou em campanhas diferentes. Em termos de OD, observou-se que os Rios Iguaçu e Belém apresentaram-se com anoxia, provavelmente, devido à grande presença de efluentes domésticos, que podem ser confirmados pelos altos valores de fósforo (P-total e ortofosfato) e formas de nitrogênio inorgânico, especialmente $\mathrm{N}$-amoniacal.

A entrada de matéria orgânica decorrente de despejos domésticos (Tabela 2, Figura 3A), em águas naturais, ocasiona diminuição da concentração de oxigênio dissolvido, prejudicando a qualidade da água (Tabela 2).

De acordo com Rostan e Cellot (1995), a absortividade (A285) determinada através da razão da absorbância em 285 $\mathrm{nm}$ pelo COD (g. $\left.\mathrm{L}^{-1}\right)$, pode ser indicativo da composição do carbono orgânico dissolvido. Valores de A285, próximos de 20 L.g ${ }^{-1}$, podem ser considerados como COD essencialmente formado por ácidos fúlvicos, enquanto que valores inferiores a 10 L.g ${ }^{-1}$ indicaram presença de carbono alifático (matéria orgânica mais lábil). Este COD mais lábil pode ser proveniente da produtividade primária ou de efluentes domésticos e/ou industriais. $\mathrm{Na}$ figura 3B, foi observado que a maioria do COD apresentou valores abaixo de $10 \mathrm{~L}_{\mathrm{g}}{ }^{-1}$, indicando carbono orgânico dissolvido mais lábil, provavelmente, de efluentes domésticos, pois além de baixo OD foram determinados valores elevados de fósforo total e N-amoniacal (Tabela 2).

O COD mais lábil também pode ser confirmado através dos espectros de emissão de fluorescência. A razão (FR) da IF emitida nos comprimentos de onda de $450 \mathrm{~nm}$ e $500 \mathrm{~nm}$ $(\mathrm{FR}=$ IF450/IF500), com excitação em 370 nm também é utilizada como indicativo de carbono orgânico mais refratário ou mais lábil. Valores de FR maiores do que 1,8 indicam fonte autóctone ou mais lábil e, valores menores do que 1,5 indicam fonte alóctone pedogênica, principalmente, ácido fúlvico. Pela Figura 3C, verifica-se que houve predomínio de COD mais lábil em alguns rios, pois os valores estiveram mais próximos ou superiores a 1,8 .

Outro aspecto que chama a atenção são os valores da intensidade de fluorescência emitida em 298 nm (Figura 3D), a qual caracteriza matéria orgânica lábil, podendo ser de esgotos domésticos (AHMAD; REYNOLDS, 1995; CHEN et al., 2002; KNAPIK; FERNANDES; AZEVEDO, 2014; PONS; BONTÉ; POTIER, 2004). Observou-se que nos períodos de menor precipitação foram maiores as intensidades de fluorescência emitidas nesta região.

Vários autores realizaram estudos para caracterizar e identificar a matéria orgânica natural de diferentes origens (FRIMMEL, 1998; PEURAVUORI; KOIVIKKO; PIHLAJA, 2002), ou para diferenciar a matéria orgânica de origem natural da matéria orgânica dissolvida proveniente de efluentes domés-

Tabela 2 - Valores médios do oxigênio dissolvido (OD), fósforo total (P-total), ortofosfato (P-PO $\left.{ }_{4}^{3-}\right), \mathrm{N}$-nitrito, N-nitrato, $\mathrm{N}$-amoniacal e COD, concentrações em mg. $\mathrm{L}^{-1}$

\begin{tabular}{lrrrrrrr}
\hline Rio & OD & P-total & ${\mathrm{P}-\mathrm{PO}_{4}{ }^{3-}}^{-}$ & Nitrito $^{-}$ & N-amoniacal & Nitrato & COD \\
\hline Iraí & 5.77 & 0.73 & 0.58 & 0.13 & 8.57 & 0.50 & 9.87 \\
& $(2.40)$ & $(0.16)$ & $(0.31)$ & $(0.03)$ & $(3.61)$ & $(0.31)$ & $(11.15)$ \\
Barigui & 4.05 & 1.21 & 0.43 & 0.17 & 4.42 & 1.31 & 14.28 \\
& $(1.54)$ & $(1.08)$ & $(0.50)$ & $(0.04)$ & $(4.38)$ & $(0.36)$ & $(11.98)$ \\
Iguaçu & 1.92 & 2.00 & 0.77 & 0.07 & 13.39 & 0.38 & 12.46 \\
& $(1.49)$ & $(1.08)$ & $(0.39)$ & $(0.03)$ & $(8.09)$ & $(0.27)$ & $(6.70)$ \\
Belém & 0.46 & 4.81 & 1.90 & 0.07 & 23.49 & 0.14 & 19.01 \\
& $(0.54)$ & $(3.15)$ & $(1.15)$ & $(0.01)$ & $(14.67)$ & $(0.06)$ & $(12.65)$ \\
Atuba & 4.06 & 3.89 & 1.84 & 0.13 & 25.91 & 0.78 & 18.59 \\
& $(1.86)$ & $(2.86)$ & $(1.71)$ & $(0.07)$ & $(20.72)$ & $(0.62)$ & $(10.04)$ \\
\hline
\end{tabular}



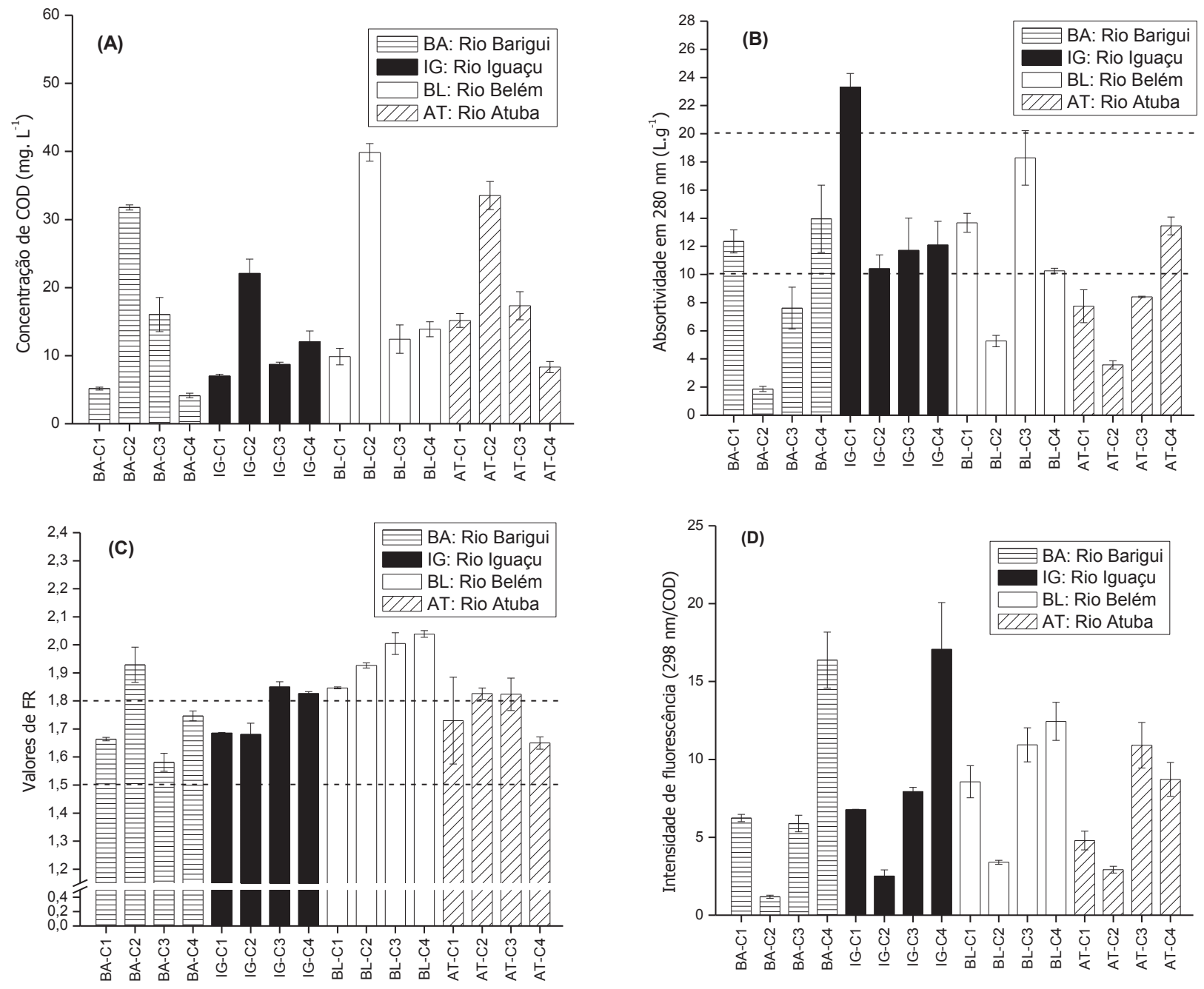

Figura 3 - Variação do COD (A), da absortividade (A285), da razão FR e da IF emitida em 298 nm nos Rios Barigui (N = 48), Iguaçu (N = 24), Atuba $(\mathrm{N}=24)$ e Belém $(\mathrm{N}=24)$ nas quatro campanhas realizadas $(\mathrm{C} 1, \mathrm{C} 2, \mathrm{C} 3$ e C4)

ticos (AHMAD; REYNOLDS, 1995; CHEN et al., 2002; 2002; PONS; BONTÉ; POTIER, 2004). Assim, considerando que a matéria orgânica dissolvida nos rios da Região Metropolitana de Curitiba apresenta tendência de ser COD de origem provenientes de efluentes de domésticos, foram analisados alguns fármacos nestes ambientes aquáticos.

$\mathrm{Na}$ tabela 3, constam os resultados, em termos de detecção, de três fármacos analisados (ibuprofeno, diclofenaco e paracetamol) nas amostras de água e de sedimento. Esta detecção confirma a entrada de matéria orgânica mais lábil proveniente de efluentes domésticos nos rios da Região Metropolitana de Curitiba.

Os rios Atuba e Belém se destacam por ter presença constante dos três fármacos em suas águas. O rio Atuba se caracteriza pela entrada do efluente da ETE Atuba Sul (AT2) e densidade populacional na região (AT1) o que pode estar ajudando na frequência desses compostos no rio Atuba, porém, apenas o ibuprofeno não foi detectado no ponto anterior à ETE
Tabela 3 - Frequência de detecção, em porcentagem (\%), dos compostos determinados, nos pontos de cada rio amostrado.

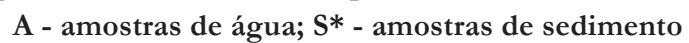

\begin{tabular}{|c|c|c|c|c|c|c|c|}
\hline \multirow{2}{*}{ Rios } & \multirow{2}{*}{ Pontos } & \multicolumn{2}{|c|}{ IBU } & \multicolumn{2}{|c|}{ PAR } & \multicolumn{2}{|c|}{ DIC } \\
\hline & & $\mathbf{A}$ & $\mathrm{S}$ & A & $\mathrm{S}$ & $\mathbf{A}$ & $\mathrm{S}$ \\
\hline \multirow{2}{*}{ Atuba } & AT1 & 50 & 0 & 100 & 0 & 100 & 0 \\
\hline & AT2 & 100 & 0 & 100 & 0 & 100 & 0 \\
\hline \multirow{4}{*}{ Barigui } & BA1 & 0 & 0 & 75 & 0 & 75 & 0 \\
\hline & BA2 & 0 & 0 & 75 & 33 & 50 & 0 \\
\hline & BA3 & 25 & 0 & 75 & 66 & 75 & 0 \\
\hline & BA4 & 0 & 0 & 100 & 0 & 100 & 0 \\
\hline \multirow{2}{*}{ Iguaçu } & IG1 & 50 & 0 & 100 & 33 & 100 & 0 \\
\hline & IG2 & 50 & 0 & 100 & 66 & 100 & 0 \\
\hline \multirow{2}{*}{ Belém } & BL1 & 75 & 0 & 100 & 33 & 100 & 0 \\
\hline & BL2 & 75 & 0 & 100 & 33 & 100 & 0 \\
\hline Iraí & IR1 & 0 & 0 & 100 & 0 & 50 & 0 \\
\hline
\end{tabular}

* Nos sedimentos foram realizadas análises em 3 campanhas. 
Kramer et al.:Determinação de anti-inflamatórios na água e sedimento e suas relações com a qualidade da água na bacia do Alto Iguaçu, Curitiba-PR.

(AT1), os demais todos tiveram 100\% de frequência antes da descarga da ETE. Já nos seus sedimentos, não foi detectada a presença de nenhum dos três compostos.

A elevada frequência no rio Belém pode estar associada à densa urbanização que afeta as margens desse rio, causando alterações na qualidade da água, com a presença de grande quantidade de esgotos domésticos.

Os rios Barigui e Iraí foram os que apresentaram as menores frequências e, portanto, indicam ter um menor impacto por esses compostos provenientes de efluentes domésticos. Este resultado está de acordo com os valores um pouco mais elevados de OD e menores de $\mathrm{N}$-amoniacal e ortofosfato, em relação aos rios Atuba, Belém e Iguaçu, apesar de serem valores elevados.

O rio Iguaçu teve uma presença constante de paracetamol e diclofenaco, mas o que se destacou foi a presença de paracetamol no sedimento (33\% no ponto IG1 e $66 \%$ no ponto IG2).

O ibuprofeno foi o fármaco com o menor índice de detecção e quantificação entre os compostos analisados, em grande parte das amostragens teve suas concentrações abaixo do limite de detecção (Figura 4).

Apenas na primeira campanha foi possível quantificar o ibuprofeno nos rio Atuba e Belém (Figura 4). No rio Atuba, a concentração encontrada foi de 102 ng. $L^{-1}$ e no Rio Belém o valor médio $(\mathrm{N}=24)$ foi de 370 ng. $\mathrm{L}^{-1}$. Em algumas amostras foi detectado, mas não foi quantificado.

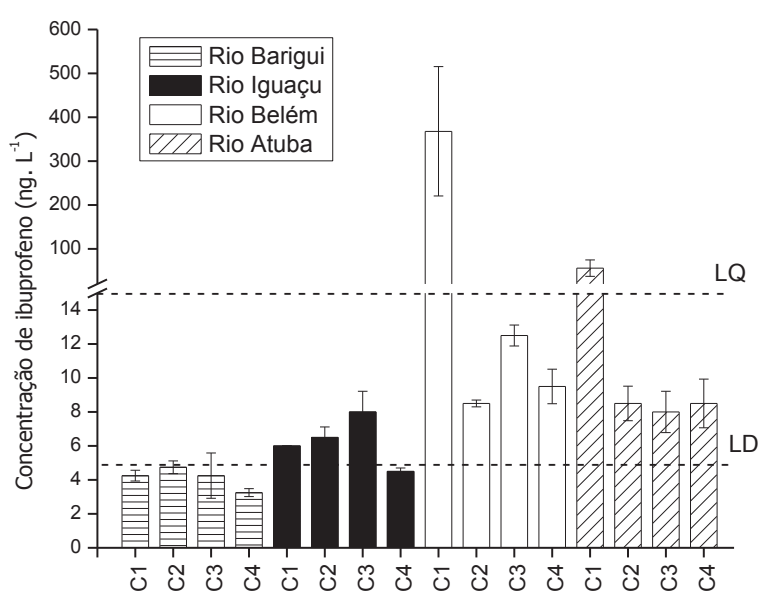

Figura 4 - Concentração de ibuprofeno na água dos Rios Barigui $(\mathrm{N}=48)$, Iguaçu $(\mathrm{N}=24)$, Atuba $(\mathrm{N}=24)$ e Belém $(\mathrm{N}=24)$ nas quatro campanhas realizadas (C1, C2, C3 e C4). O LD e o LQ da água foram 10 e $31 \mathrm{ng} . \mathrm{L}^{-1}$

A baixa frequência de detecção do ibuprofeno já era esperada, devido ao baixo consumo desse medicamento no Brasil, o que também pode ser confirmados por outros estudos realizados. Em Campinas (MONTAGNER; JARDIM, 2011) e no Rio de Janeiro (STUMPF et al., 1999), este fármaco também foi detectado.

Em outros países, o ibuprofeno é mais consumido e, segundo Ternes (1998), na Alemanha, no ano de 1995, foi prescrito um total de 105 toneladas desse componente. Ternes
(1998) afirma ainda ter encontrado na saída de uma das ETE estudadas 370 ng. $L^{-1}$ e 70 ng.. L $^{-1}$ em um dos rios da região. Já López-Serna et al. (2013), na bacia do Rio Guadalquivir, no sul da Espanha, encontraram valores médios de aproximadamente 600 ng.L- ${ }^{-1}$. Em Portugal, Paíga et al. (2013), no rio Lima, foi quantificado 723 ng. $\mathrm{L}^{-1}$, sendo que em aproximadamente $50 \%$ dos pontos analisados foi detectado o ibuprofeno. Os resultados desses estudos corroboram com os resultados obtidos RMC.

A não detecção de ibuprofeno nos sedimentos se deve, principalmente, ao fato de ser uma molécula química hidrofílica e, segundo trabalho experimental realizado por Loffler et al. (2005), o ibuprofeno possui uma meia vida de 8 dias em ambientes aquáticos e não é encontrado em sedimentos e nem seu metabolito principal, o 2-hidroxi-ibuprofeno.

O diclofenaco apresentou a peculiaridade de ter sido detectado com frequência na água, porém no sedimento não foi detectado em nenhuma das três amostragens. Esse fato pode estar relacionado ao seu valor de $\log$ Kow $(4,51)$, que mostra uma maior tendência de estar presente na coluna d'água e não incorporada nos sedimentos. Até mesmo na água em muitos dos pontos amostrados ficou abaixo dos limites de quantificação (Figura 5), o que pode indicar menor consumo desde fármaco pela população local ou degradação. Essa degradação foi constatada no estudo de Buser, Poiger e Muller (1998), que observaram, em lagos naturais, que $90 \%$ do diclofenaco foi fotodegradado.

A terceira amostragem (C3) apresentou as maiores concentrações de diclofenaco nos rios analisados, especialmente no rio Iguaçu, com valores de 285 ng. $\mathrm{L}^{-1}$. No rio Barigui, foi quantificado diclofenaco na primeira campanha, porém somente no ponto BA4 que fica na entrada do município de Araucária. O rio Atuba possui a peculiaridade do efluente da ETE e isso ficou evidente na concentração de diclofenaco após jusante da mesma, pois a concentração na terceira amostragem foi maior no ponto próximo da saída da estação (AT2, 54 ng..-1 ${ }^{-1}$ em relação a montante da ETE (AT1, 43 ng. $\mathrm{L}^{-1}$ ) e, na quarta amostragem (C4) somente no ponto AT2 foi quantificada diclofenaco (34 ng..- $\left.{ }^{-1}\right)$. No rio Atuba, o diclofenaco correlacionou-se positivamente

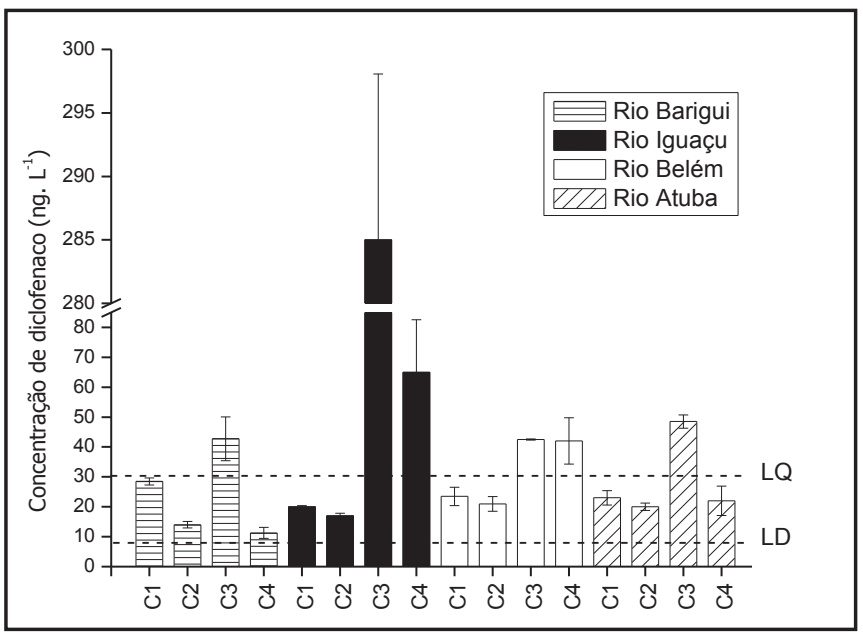

Figura 5 - Concentração de diclofenaco na água dos Rios Barigui $(\mathrm{N}=48)$, Iguaçu $(\mathrm{N}=24)$, Atuba $(\mathrm{N}=24)$ e Belém $(\mathrm{N}=24)$ nas quatro campanhas realizadas (C1, C2, C3 e C4). O LD de 9 ng. $\mathrm{L}^{-1}$ e $L Q$ de 31.ng $\mathrm{L}^{-1}$ 
com o ortofosfato $\left(r_{s}=0,6078 ; \mathrm{N}=24\right)$ e com a intensidade de fluorescência em $298 \mathrm{~nm}\left(r_{s}=0,7306 ; \mathrm{N}=24\right)$ indicando a possível influência do efluente da ETE.

O rio Belém também apresentou diclofenaco, principalmente na C4, com concentração de 61 ng. $\mathrm{L}^{-1}$. Neste rio, as correlações encontradas com o diclofenaco, novamente sugerem a influência por esgoto doméstico $\left(\mathrm{Cl}^{-}: r_{S}=0,8107 ; \mathrm{N}=18\right.$; $\mathrm{N}$-amoniacal: $\left.r_{s}=0,5674 ; \mathrm{N}=18\right)$.

Estudo realizado na cidade do Rio de Janeiro por Stumpf et al. (1999) e em Campinas por Montagner e Jardim (2011) corroboram com os resultados obtidos nos rios da RMC. No Rio de Janeiro, foi encontrada a concentração de 60 ng.L. ${ }^{-1}$ e em Campinas, de 115 ng.L ${ }^{-1}$. Já na Europa, as concentrações encontradas são superiores, especialmente na Alemanha, aonde a quantificação nos rios chegou a 600 ng.L L $^{-1}$ (Ternes, 1998), que segundo o mesmo autor está relacionado com o consumo desse medicamento, pois em 1995 foram prescritas 75 toneladas de diclofenaco.

O paracetamol foi um dos fármacos estudados que apresentou as maiores ocorrências e concentrações, tanto no ambiente aquático (Figura 6A) como nos sedimentos (Figura 6B).
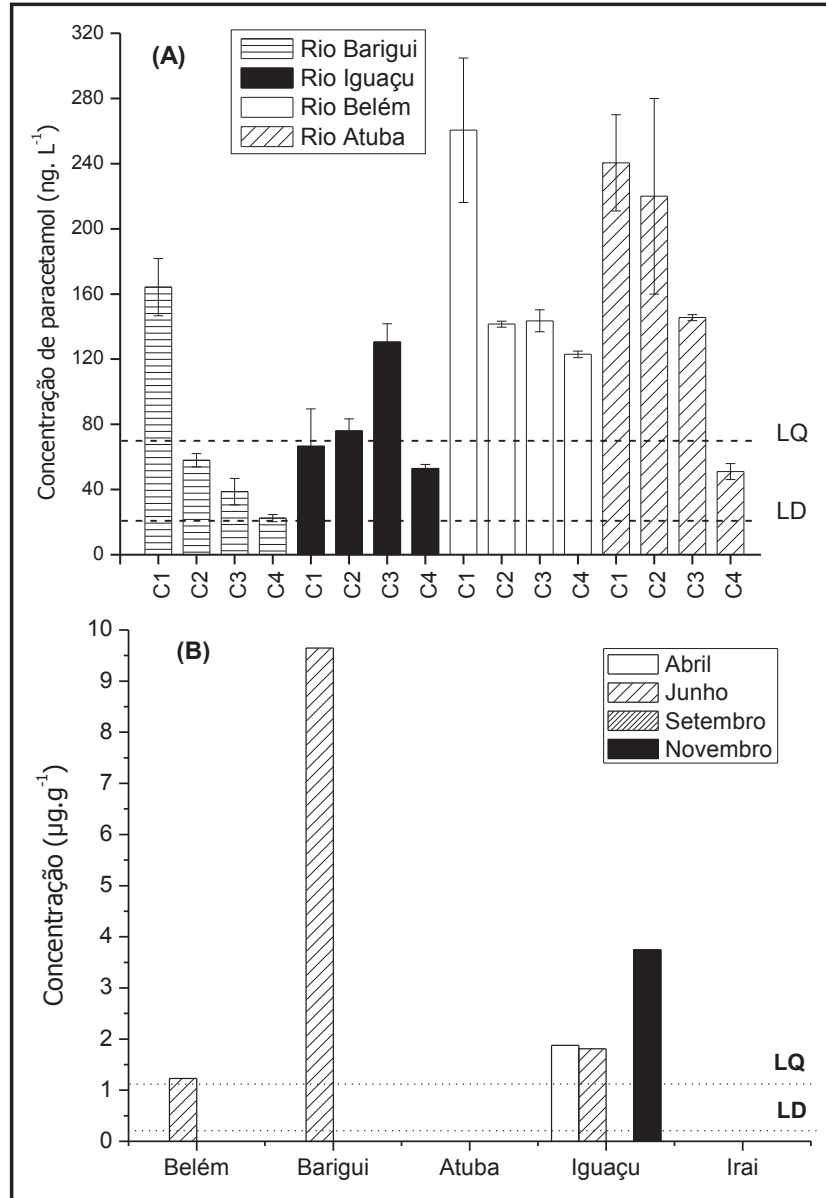

Figura 6 - Concentração de paracetamol na água (A) nos Rios Barigui $(\mathbf{N}=48)$, Iguaçu $(\mathrm{N}=24)$, Atuba $(\mathbf{N}=24)$ e Belém $(\mathrm{N}=24)$ nas quatro campanhas realizadas $(\mathrm{C} 1, \mathrm{C} 2, \mathrm{C} 3$ e $\mathrm{C} 4)$ e no sedimento (B). O LD e o LQ da água foi 22 e $73 \mathrm{ng} \mathrm{L}^{-1}$, respectivamente, e para o sedimento o $L D$ e $L Q$ foram 0,38 e $1,26 \mu \mathrm{g} \mathrm{g}^{-1}$, respectivamente

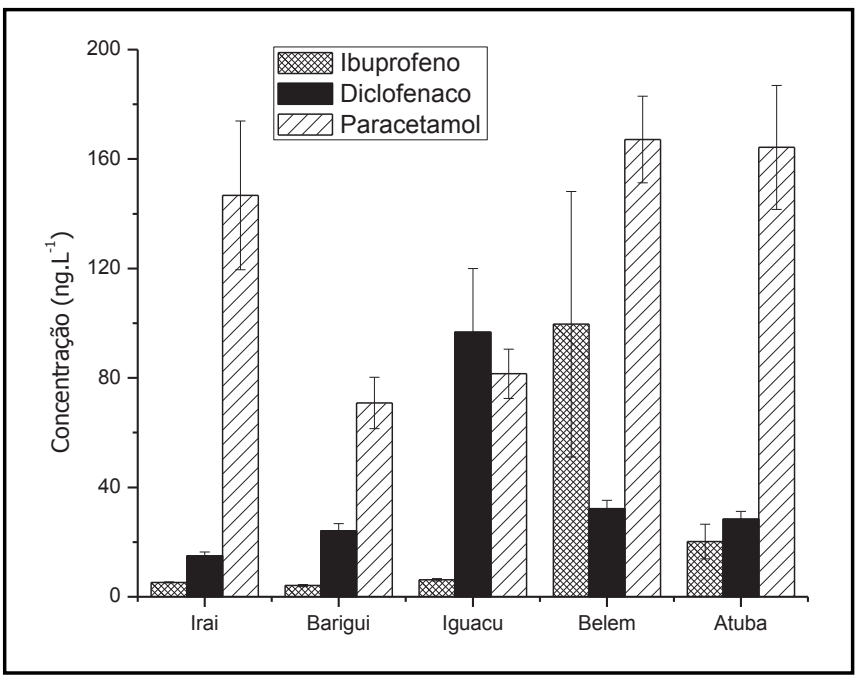

Figura 7 - Valores médios dos fármacos determinados nos Rios Barigui ( $\mathbf{N}=48)$, Iguaçu $(\mathrm{N}=24)$, Atuba $(\mathrm{N}=24)$ e Belém $(\mathrm{N}=24)$ nas quatro campanhas realizadas $(\mathrm{C} 1, \mathrm{C} 2, \mathrm{C} 3$ e $\mathrm{C} 4)$. O LD de 9 ng. $L^{-1}$ e LQ de 31.ng $L^{-1}$

Grande parte dos rios apresentaram tendências semelhantes na concentração de paracetamol na água (Figura 6A), tendo a primeira campanha com maiores concentrações (C1), seguida da terceira (C3), da segunda (C2) e por fim da quarta campanha (C4). O rio Iraí (Figura 7) foi discrepante nessa análise, pois teve um ápice de concentração de 367 ng. $\mathrm{L}^{-1}$.

O rio Belém e o Rio Atuba apresentaram mais impactados por efluentes domésticos devido a incidência de fármacos nas amostragens realizadas (Figura 7), chegando a ter 261 ng. L $^{-1}$ (C1) de paracetamol no Rio Belém (Figura 6). Estas maiores concentrações foram obtidas no período que teve as menores precipitações locais. Por mais altas que pareçam as concentrações de paracetamol encontradas nesses rios, ainda assim ficaram abaixo das encontradas por Montagner e Jardim (2011) na cidade de Campinas, que chegou ao valor 13440 ng.L ${ }^{-1}$ na bacia hidrográfica do Atibaia.

Loffler et al. (2005) afirmaram que o paracetamol é totalmente transformado em 14 dias na água e quando vai para o sedimento se transforma em um produto não extraível e, portanto, não detectado em altas concentrações. Os rios Barigui e Iguaçu apresentaram concentrações de paracetamol no sedimento, podendo ser um indicativo de contaminação recente de efluentes domésticos. No rio Iguaçu, a detecção de paracetamol no sedimento foi de 1,88 $\mu \mathrm{g} \cdot \mathrm{g}^{-1}$ na C1, de 1,81 $\mu \mathrm{g} \cdot \mathrm{g}^{-1}$ na C2 e de $3,74 \mu \mathrm{g} \cdot \mathrm{g}^{-1}$ na C4. Nesse mesmo rio, foi observada uma correlação negativa entre as concentrações de paracetamol na água e no sedimento $(r=-0,52 ; p<0,046 ; N=15)$ o que indica que pode estar havendo uma sorção desse fármaco no sedimento.

\section{CONCLUSÃO}

Foi possível detectar e quantificar os três produtos farmacêuticos propostos neste trabalho, tendo como destaque, o paracetamol que foi quantificado em todos os pontos em pelo menos uma das amostragens de água realizada. O diclofenaco 
Kramer et al.:Determinação de anti-inflamatórios na água e sedimento e suas relações com a qualidade da água na bacia do Alto Iguaçu, Curitiba-PR.

também foi detectado nos rios, porém em menores concentrações e frequência, ao contrário do ibuprofeno que foi quantificado na primeira amostragem em dois dos rios analisados. Esses contaminantes aportam nos rios, na maior parte das vezes, pela entrada de esgotos clandestinos ou não vindos de regiões de invasão das margens dos rios.

O ibuprofeno e o diclofenaco não foram quantificados nos sedimentos por não apresentar efeito acumulativo nesta matriz pelo seu Kow, ou seja, por serem hidrofílicos, sendo quantificados na forma dissolvida.

Os rios Iguaçu, Atuba e Belém se destacaram negativamente com a forte presença de contaminantes provenientes de esgotos domésticos e os rios Barigui e Iraí, também apresentam contaminação de mesma fonte mas com níveis menores de deterioração.

De um modo geral essa contaminação acontece não só pelo consumo desses fármacos, mas principalmente pela entrada de efluentes domésticos in natura ou tratados de forma ineficiente.

\section{AGRADECIMENTOS}

Ao CNPq pelo financiamento (Processo474900/2011-8); a Fundação Araucária (Bolsa PQ); a CAPES pelo apoio através de bolsa de estudos, á UTFPR e ao CT-Infra 2010/FINEP pelos Recursos FNDCT, subprojeto NIPTA -Núcleo Interdisciplinar de Pesquisa em Tecnologias Ambientais.

\section{REFERÊNCIAS}

AHMAD, S. R.; REYNOLDS, D. M. Synchronous fluorescence spectroscopy of wastewater and some potential constituents. Water Res., v. 29, n. 6, p. 1599-1602, June 1995.

ANDREOLI, C. V.; DALARMI, D.; LARA, A. I.; ANDREOLI, F. N. Limites ao desenvolvimento da região metropolitana de Curitiba, impostos pela escassez de água. In: SILUBESA SIMPÓSIO BRASILEIRO DE ENGENHARIA SANITÁRIA E AMBIENTAL, 9., 2000, Porto Seguro. Anais... Porto seguro: [s.n.], 2000. p. 185-195.

APHA - American Public Health Association, American Water Works Association, Water Environment Federation. Standard Methods for the Examination of Water and Wastewater. 22th ed. Washington, DC: American Public Health Association, American Water Works Association, Water Environment Federation, 2012.

AQUINO, S. F.; BRANDT, E. M. F.; CHERNICHARO, C. A. L. Remoção de fármacos e desreguladores endócrinos em estações de tratamento de esgoto: revisão da literatura. Eng. Sanit. Ambient., v. 18, n. 3, p. 187-204, jul./set. 2013.

AZEVEDO, J. C. R.; NOZAKI, J. Análise de fluorescência de substâncias húmicas extraídas da água, solo e sedimento da Lagoa dos Patos/MS. Quím. Nova, v. 31, n. 6, p. 1324-1329, 2008.
BUSER, H. R.; POIGER, T.; MULLER, M. D. Occurrence and fate of the pharmaceutical drug diclofenac in surface waters: Rapid photodegradation in a lake. Environ. Sci. Technol.,v. 32, n. 22, p. 3449-3456, Set. 1998.

CHEN, J.; GU, B. H.; LEBOUF, E. J.; PAN, H. J.; DAI, S. Spectroscopic characterization of the structural and functional properties of natural organic matter fractions. Chemosphere, v. 48 , n. 1 , p. 59-68, July 2002.

CHEN, W.; WESTERHOFF, P.; LEENHEER, J. A.; BOOKSH, $\mathrm{K}$. Fluorescence excitation-emission matrix regional integration to quantify spectra for dissolved organic matter. Environ. Sci. Technol., v. 37, n. 24, p. 5701-5710, Dec. 2003.

CHRISTOFARO, C.; LEAO, M. M. D. Tratamento de dados censurados em estudos ambientais. Quim. Nova, v. 37, n. 1, p. 104-110, 2014.

DAUGHTON, C. G.; TERNES, T. A. Pharmaceuticals and personal care products in the environment: agents of subtle change? Environ. Health Perspect., v. 107, p. 907-938, Dec. 1999. Supplement 6 .

EICKHOFF, P.; HEINECK, I.; SEIXAS, L. J. Gerenciamento e destinação final de medicamentos: uma discussão sobre o problema. Rev. Bras. Farmácia, v. 90, n. 1, p. 64-68, 2009.

EMERENCIANO, D. P.; SILVA, H. F. O.; CARVALHO, G. C.; SOUZA, J. M.; CRUZ, M. F. C.; RIBEIRO, L. P. D.; MOURA, F. $\mathrm{V}$. Controle de qualidade de medicamentos contendo diclofenaco sódico e potássico utilizando a espectroscopia no Infravermelho próximo NIR. In: CONGRESSO BRASILEIRO DE QUÍMICA, 48., 2008, Rio de Janeiro. Anais... [S.l.: s.n.], 2008.

FATTA-KASSINOS, D.; MERIC, S.; NIKOLAOU, A. Pharmaceutical residues in environmental waters and wastewater: current state of knowledge and future research. Anal. Bioanal. Chem., v. 399, n. 1, p. 251-275, Jan. 2011.

FRIMMEL, F. H. Characterization of natural organic matter as major constituents in aquatic systems. J. Contaminant Hydrol., v. 35, n. 1-3, p. 201-216, Dec. 1998.

KASPRZYK-HORDERN, B. Pharmacologically active compounds in the environment and their chirality. Chem. Soc. Rev., v. 39, n. 11, p. 4466-4503, Nov. 2010.

KASPRZYK-HORDERN, B.; DINSDALE, R. M.; GUWY, A. J. Illicit drugs and pharmaceuticals in the environment - Forensic applications of environmental data. Part 1: Estimation of the usage of drugs in local communities. Environ. Pollut., v. 157, n. 6, p. 1773-1777, June 2009.

KNAPIK, H. G.; FERNANDES, C. V. S; AZEVEDO, J. C. R. Aplicabilidade de técnicas de espectroscopia e da concentração de carbono orgânico dissolvido na caracterização da matéria 
orgânica em rios urbanos. RBRH: revista brasileira de recursos hídricos, v. 19, n. 4, p. 214-225, out./dez. 2014.

KNAPIK, H. G.; FRANÇA, M. S; FERNANDES, C. V. S.; MASINI, L. S.; MARIN, M. C. F. C.; PORTO, M. F. A. Análise crítica da calibração do modelo de qualidade de água QUAL2E - estudo de caso da bacia do Alto Iguaçu. REGA, v. 5, n. 2, p. 25-37, jul./dez. 2008.

KUMMERER, K. Pharmaceuticals in the Environment. Annu. Rev. Environ. Resour., v. 35, p. 57-75, 2010.

LA FARRE, M.; PEREZ, S.; KANTIANI, L.; BARCELO, D. Fate and toxicity of emerging pollutants, their metabolites and transformation products in the aquatic environment. Trends Anal. Chem., v. 27, n. 11, p. 991-1007, Dec. 2008.

LOFFLER, D.; ROMBKE, J.; MELLER, M.; TERNES, T. A. Environmental fate of pharmaceuticals in water/sediment systems. Environ. Sci. Technol., v. 39, n. 14, p. 5209-5218, July 2005.

LOPEZ-SERNA, R.; KASPRZYK-HORDERN, B.; PETROVIC, M.; BARCELO, D. Multi-residue enantiomeric analysis of pharmaceuticals and their active metabolites in the Guadalquivir River basin (South Spain) by chiral liquid chromatography coupled with tandem mass spectrometry. Anal. Bioanal. Chem., v. 405, n. 18, p. 5859-5873, July 2013.

MA, H.; ALLEN, H. E.; YIN, Y. Characterization of isolated fractions of dissolved organic matter from natural waters and a wastewater effluent. Water Res., v. 35, n. 4, p. 985-996, Mar. 2001.

MONTAGNER, C. C.; JARDIM, W. F. Spatial and Seasonal Variations of Pharmaceuticals and Endocrine Disruptors in the Atibaia River, Sao Paulo State (Brazil). J. Bra\%. Chem. Soc.,v. 22, n. 8, p. 1452-1462, Aug. 2011.

OLIVEIRA, J. L.; BOROSKI, M.; AZEVEDO, J. C. R.; NOZAKI, J. Spectroscopic investigation of humic substances in a tropical lake during a complete hydrological cycle. Acta Hydrochim. Hydrobiol., v. 34, n. 6, p. 608-617, Dec. 2006.

ONESIOS, K. M.; YU, J. T.; BOUWER, E. J. Biodegradation and removal of pharmaceuticals and personal care products in treatment systems: a review. Biodegradation, v. 20, n. 4, p. 441466, July 2009.

PAÍGA, P.; SANTOS, L. H. M. L. M.; AMORIM, C. G.; ARAÚJO, A. N.; MONTENEGRO, M. C. B. S. M.; PENA, A.; DELERUEMATOS, C. Pilot monitoring study of ibuprofen in surface waters of north of Portugal. Environ. Sci. Pollut. Res. Int., v. 20, n. 4, p. 2410-2420, Apr. 2013.

PEURAVUORI, J.; KOIVIKKO, R.; PIHLAJA, K. Characterization, differentiation and classification of aquatic humic matter separated with different sorbents: synchronous scanning fluorescence spectroscopy. Water Res., v. 36, n. 18, p.
4552-4562, Nov. 2002

PONS, M.; BONTÉ, S. L.; POTIER, O. Spectral analysis and fingerprinting for biomedia characterization. J. Biotechnol., v. 113, n. 1-3, p. 211-230, Sept. 2004.

RICHARDSON, M. L.; BOWRON, J. M. The Fate of Pharmaceutical Chemicals in the Aquatic Environment. J. Pharm. Pharmacol., v. 37, n. 1, p. 1-12, Jan. 1985.

ROSTAN, J. C.; CELLOT, B. On the use of spectrophotometry to assess dissolved organic carbon origin variations in the Upper Rhône River. Aquatic Sci., v. 57, n. 1, p. 1015-1621, 1995.

SCHNELL, S.; BOLS, N. C.; BARATA, C.; PORTE, C. Single and combined toxicity of pharmaceuticals and personal care products (PPCPs) on the rainbow trout liver cell line RTL-W1. Aquat. Toxicol., v. 93, n. 4, p. 244-252, July 2009.

SEMA. Bacias hidrográficas do Paraná: sede histórica. Curitiba: SEMA, 2010.

SENESI, N. Molecular and quantitative aspects of the chemistry of fulvic acid and its interactions with metal ions and organic chemicals. Part II: The fluorescence spectroscopy approach. Anal. Chim. Acta, v. 232, p. 77-106, 1990.

SHIN, H.; OH, J. Simultaneous determination of non-steroidal anti-inflammatory drugs in river water by gas chromatographymass spectrometry. J. Sep. Sci., v. 35, n. 4, p. 541-547, Feb. 2012.

SILVA, B. F.; JELIC, A.; LÓPEZ-SERNA, R.; MOZETO, A. A.; PETROVIC, M.; BARCELÓ, D. Occurrence and distribution of pharmaceuticals in surface water, suspended solids and sediments of the Ebro river basin, Spain. Chemosphere, v. 85, n. 8, p. 1331-1339, Nov. 2011

STUMPF, M.; TERNES, T. A.; WILKEN, R.; RODRIGUES, S. V.; BAUMANN, W. Polar drug residues in sewage and natural waters in the state of Rio de Janeiro, Brazil. Sci. Total Environ., v. 225, n. 1-2, p. 135-141, Jan. 1999.

TERNES, T. A. Occurrence of drugs in German sewage treatment plants and rivers. Water Res., v. 32, n. 11, p. 32453260, Nov. 1998.

WESTERHOFF, P.; ANNING, D. Concentrations and characteristics of organic carbon in surface water in Arizona: influence of urbanization. J. Hydrol., v. 236, n. 3-4, p. 202-222, Sept. 2000.

XU, J.; WU, L. S.; CHEN, W. P.; CHANG, A. C. Simultaneous determination of pharmaceuticals, endocrine disrupting compounds and hormone in soils by gas chromatography-mass spectrometry. J. Chromatogr. A, v. 1202, n. 2, p. 189-195, Aug. 2008.

YAMAMOTO, C. R.; CANALI, N. E. Importância das wetlands 
Kramer et al.:Determinação de anti-inflamatórios na água e sedimento e suas relações com a qualidade da água na bacia do Alto Iguaçu, Curitiba-PR.

para a qualidade das águas na região Metropolitana de Curitiba

- PR. Rev. Geografar, v. 7, n. 1, p. 161-189, jun. 2012. 